\title{
Acute-on-Chronic Osteomyelitis of the Scapula Treated via Trephination
} Timothy Woodacre* and Timothy Mason

The Knee Unit, Princess Elizabeth Orthopaedic Centre, Royal Devon and Exeter Hospital, UK

"Correponding author: Timothy Woodacre, The Knee Unit, Princess Elizabeth Orthopaedic Centre, Royal Devon and Exeter Hospital, UK, Tel: +447824393322; E-mail: timothywoodacre@yahoo.co.uk

Rec date: Jun 17, 2014; Acc date: July 23, 2014; Pub date: July 25, 2014

Copyright: (c) 2014 Woodacre T, et al., This is an open-access article distributed under the terms of the Creative Commons Attribution License, which permits unrestricted use, distribution, and reproduction in any medium, provided the original author and source are credited.

\begin{abstract}
We present a 12 year old girl who developed pyrexia and a widespread rash following 5 weeks of right shoulder pain. No initial source for sepsis was found. She was diagnosed with right scapula osteomyelitis following plain radiographs and Magnetic Resonance Imaging (MRI). No collection was demonstrated. Despite five days of intravenous antibiotics, she became haemodynamically compromised. She was taken to theatre and her scapula was trephined. Whilst no gross collection was drained, post-operatively she improved rapidly and was discharged home on intravenous (IV) antibiotics.
\end{abstract}

Keywords: Osteomyelitis; Trephination; chronic inflammation

\section{Background}

Osteomyelitis is defined as the acute or chronic inflammation of bone and its surrounding structures by pathogenic organism [1]. Haematological spread is the most common method in children (staphylococcus aureus in $80 \%$ of cases2). Most commonly affected are the humerus, tibia, femur, maxilla, vertebrae and maxillary bodies. Long bones are more commonly affected in children because of their highly vascular metaphyses [2]. Involvement of short flat bones such as the scapulae is rare. Acute presentation of scapula osteomyelitis is however recognised in the literature [3], We describe a case of subacute osteomyelitis of the scapula in a child with an atypical presentation, successfully treated with trephination.

\section{Case Presentation}

We present a previously fit and well 12 year old girl who developed insidious onset right shoulder pain. This was initially attributed to straining during a vigorous game of swing ball. Shoulder pain persisted for 5 weeks before she became systemically unwell. She was admitted to the local district general hospital by the paediatric team with a 4 day history of pyrexia and a progressing erythematous papular rash on her chest. Initial observations revealed her to have a temperature spiking to $40 \mathrm{oC}$ and a tachycardia of 136 . Full systemic examination and initial investigations failed to reveal a source of infection. C-reactive protein (CRP) and neutrophil count were raised at $249 \mathrm{mg} / \mathrm{L}$ and $8.2^{\star} 109 / \mathrm{L}$ respectively. She was started on IV ceftriaxone, and later flucloxacillin, for treatment of sepsis of unknown origin. Her pyrexia persisted. Her CRP rose to $311 \mathrm{mg} / \mathrm{L}$ within 48 hours.

72 hours after admission she was reviewed by the orthopaedic team. Examination revealed no localised deformity or swelling, but tenderness to the lateral border of her right scapula. Shoulder flexion and abduction was limited to 90 degrees due to pain.

\section{Investigations}

Plain radiograph of her right shoulder revealed an irregular lateral border of the scapula (Figure 1) corresponding to her clinical findings. Subsequent MRI of her right shoulder suggested osteomyelitis of the lateral aspect of the right scapula with no surrounding collection and no evidence of septic arthritis (Figure 2).

Blood tests showed a positive antinuclear antibody (ANA) with speckled pattern and a moderately raised anti-streptolysin oxygenlabile (ASO) titre, suggestive of infection; however initial blood cultures failed to identify a pathogen.

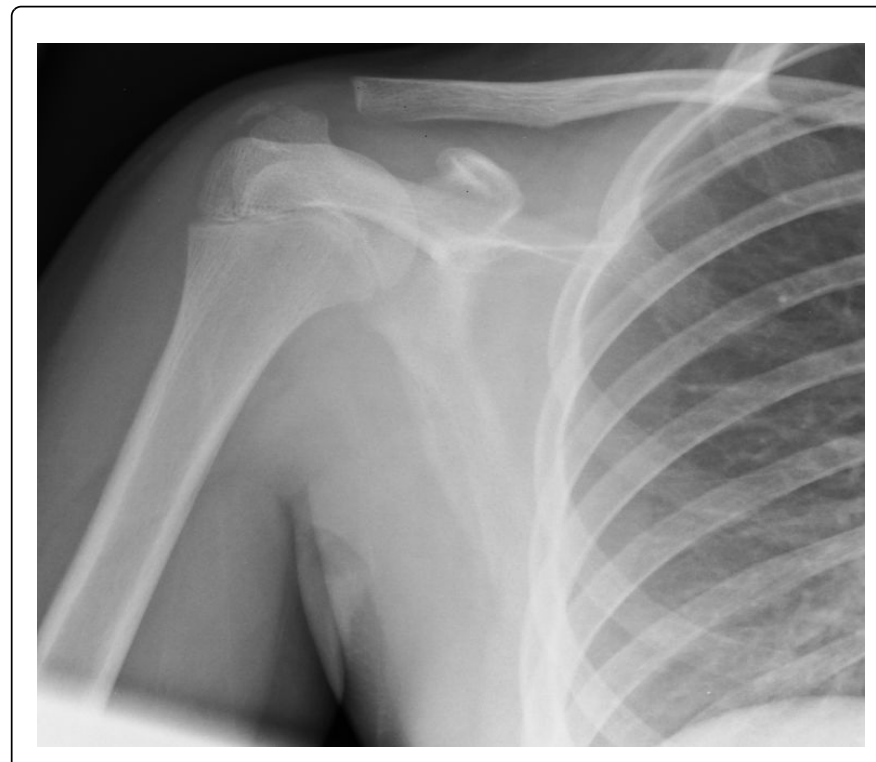

Figure 1: AP radiograph of the shoulder demonstrating irregularity of the lateral border of the scapula. 


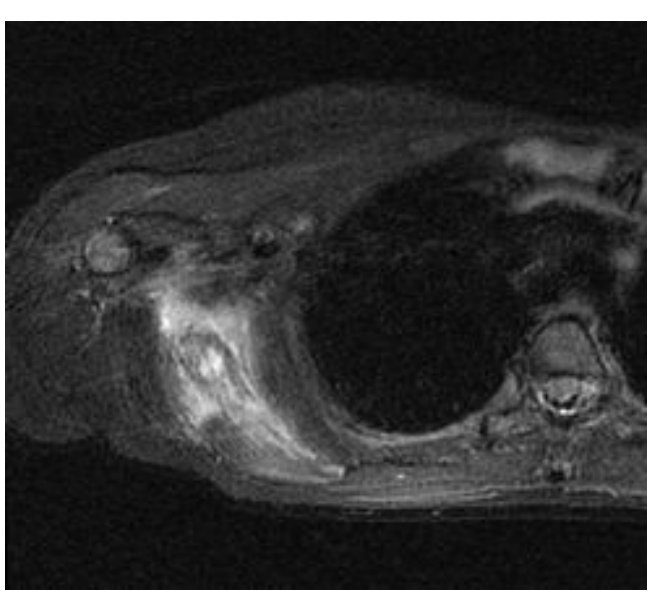

Figure 2: Axial T2 weighted MRI of the shoulder demonstrating high signal in the scapula and surrounding soft tissue

\section{Treatment}

A diagnosis of osteomyelitis of the scapula was made. Following diagnosis via MRI, she continued with a further 48 hours of IV antibiotic therapy (5 days in total). She continued to be systemically unwell. Due to her ongoing haemodynamic compromise and failure to respond to appropriate non-operative management she was taken to theatre for exploration. This revealed no localised collection of pus so the affected area of the scapula was trephined and washed out. She demonstrated an almost immediate clinical response with her pyrexia and systemic symptoms normalising. She was discharged 48hours post-surgery on outpatient IV antibiotics and concurrent monitoring of her inflammatory markers. She received a total of 4 weeks of IV antibiotics followed by 2 weeks of oral antibiotics. Clinical review 3 months post-discharge revealed full pain-free return of shoulder function and a CRP of $2 \mathrm{mg} / \mathrm{L}$.

\section{Discussion}

Osteomyelitis of scapula is relatively rare [4]. It is more common in males and in lower socio-economic class and has been reported as a complication of endocarditis [5], steroid injection [6], immunodeficiency [7] or trauma. However, other than the overuse injury during swing ball there had been no prior illness or trauma in this case. A systematic review by Koubaa et al [3] examining all reported cases of osteomyelitis over 10 years revealed only 4 to involve the scapula (an incidence of $2.6 \%$ ), a case series by Martini et al [8] revealed 2 cases from 420 (incidence of $0.5 \%$ ) and Heinonen et al reported 4 in 942 cases (0.42\%) [9].

Osteomyelitis of the scapula usually presents as an acute event [4]. Koubaa et al. demonstrated a mean presentation within $48 \mathrm{hrs}$. The presentation of this case was atypical, with shoulder pain occurring several weeks before acute systemic deterioration.There is often delay in diagnosis and treatment of osteomyelitis of the scapula due to the rarity of the disorder and generalised, non-specific symptoms. As infection progresses there is extension of the pus outside of the scapula into the soft tissues, causing functional incapacity. If ineffectually treated it can spread regionally to involve the spinal column3 or the glenohumeral joint [10] and cause overwhelming sepsis. Osteomyelitis is not usually visible on plain radiograph until 20 days of infection [11] or until there is $30 \%$ of bony destruction [12]. MRI or technetium scintigraphy can be used to aid diagnosis. MRI has a high specificity and sensitivity [13]. In the case presented, although changes were evident on radiograph, no significant collection or gross bony destruction was found on MRI or during surgical exploration.

Treatment for osteomyelitis, irrespective of location, initially involves targeted antibiotic therapy and rest for the affected area2. Trephination is proposed when there is a purulent collection within the bone3. In the case presented trephination was initiated in the absence of an obvious collection but in response to failed nonoperative management and demonstrated a successful clinical response.

\section{Learning Points}

Scapula osteomyelitis should be an important if rare differential diagnosis for shoulder pain and pyrexia.

Acute on chronic osteomyelitis without permanent disability has not previously been described in literature.

Trephination of the scapula may be indicated, even in the absence of a defined collection, when non-operative treatment has been ineffective.

\section{References}

1. http://emedicine.medscape.com/article/785020-overview.

2. Lamprecht E (1997) Acute osteomyelitis in childhood. Orthopaede 26: 868-878.

3. M Koubaa, Mnif H, Zrig M, Jawahdou R, Sahnoun N, et al (2009) Acute hematogenous osteomyelitis of the scapula in children. Orthopaedics \& Traumatology: Surgery \& Research 632-635.

4. Williams GA (1927) Osteomyelitis of the scapula: report of a case. JBone Joint Surg Am 9: 308-309.

5. Mertens J, Van Gaever V, Ham H, Van De Wiele C (2010) Scapular osteomyelitis as a rare complication of staphylococcus aureus endocarditis: bone scintigraphy findings, Clin Nucl Med. 35: 595-597.

6. Buckley SL, Alexander AH, Barrack RL (1989) Scapular osteomyelitis an unusual complication following subacromial corticosteroid injection Orthop Rev 18: 321-324.

7. Sorensen RU, Boehm KD, Kaplan D, Berger M (1992) Cryptococcal osteomyelitis and cellular immunodeficiency associated with interleukin-2 deficiency. The Journal of Paediatrics 121: 873-879

8. M Martini, Daoud A, Saïghi-Bouaouina A, F Ziani, F Burny, et al (1994) Traitement chirurgical des ostéomyélites hématogènes chroniques. Une série de 420 cas, Rev Chir Orthop 80: 642-650.

9. Heinonen J (1925) Acta chir. Scandinav.LVIII, 289, 1924. (mt. Abs. Surg., XLI, 308

10. Obeidat MM, Omari A (2010) Osteomyelitis of the scapula with secondary septic arthritis of the shoulder joint. Singapore Med J 51: e1

11. http://www.ncbi.nlm.nih.gov/pubmed/13450575

12. Faden H, Grossi M (1991) Acute osteomyelitis in children. Reassessment of etiologic agents and their clinical characteristics. Am J Dis Child 145: 65-69.

13. Jaramillo D, Kim J (2011) Imaging of Acute Hematogenous Osteomyelitis and Septic Arthritis in Children and Adults. Evidence based imaging 287-308. 